\title{
THE RICCATI EQUATION METHOD WITH \\ VARIABLE EXPANSION COEFFICIENTS. III. SOLVING THE NEWELL-WHITEHEAD EQUATION
}

\author{
SOLOMON M. ANTONIOU
}

Abstract. The Riccati equation method with variable expansion coefficients, introduced in previous papers, is used to find traveling wave solutions to the Newell-Whitehead (NW) equation $u_{t}=u_{x x}+a u-b u^{3}$. The $\xi$-dependent coefficients $A$ and $B$ of the Riccati equation $Y^{\prime}=A+B Y^{2}$ are either proportional each other or their product is equals to an exponential function. They are determined as solutions of ODEs they satisfy and their solutions are expressed either in terms of Bessel's functions or in terms of functions already found in Paper I. The same situation occurs for the expansion coefficients as well. The function $Y$ which is a solution of Riccati's equation, is expressed in terms of Bessel functions or it is a constant quantity.

Mathematics subject classification (2010): 35C05, 35C07, 35K57.

Keywords and phrases: Riccati equation method, nonlinear evolution equations, traveling wave solutions, Newell-Whitehead equation, Bessel functions, exact solutions.

\section{REFERENCES}

[1] P. C. Abbott, E. J. Parkes and B. R. Duffy, The Jacobi elliptic-function method for finding periodicwave solutions to nonlinear evolution equations, Available online at http//physics.uwa.edu.au/pub/Mathematica/Solitons .

[2] M. A. Abdou, Adomian decomposition method for solving the telegraph equation in charged particle transport, J. Quant. Spectro. Rad. Trans., 95 (2005), 407-414.

[3] M. A. Abdou, Exact solutions for nonlinear evolution equations via the extended projective Riccati equation expansion method, Electr. J. Theor. Phys., 4 (2007), 17-30.

[4] M. A. Abdou, The extended F-expansion method and its applications for a class of nonlinear evolution equations, Chaos, Solitons and Fractals, 31 (2007), 95-104.

[5] M. A. Abdou, On the variational iteration method, Phys. Lett. A, 366 (2007), 61-68.

[6] M. A. Abdou, A generalized auxiliary equation method and its applications, J. Nonl. Dyn., 52 (2008), 95-102.

[7] M. A. Abdou, An improved generalized F-expansion method and its applications, J. Comp. Appl. Math., 214 (2008), 202-208.

[8] M. A. Abdou, Generalized solitary and periodic solutions for nonlinear partial differential equations by the exp-function method, J. Nonl. Dyn., 52 (2008), 1-9.

[9] M. A. Abdou and A. A. Soliman, New applications of Variational Iteration Method, Physica D, 211 (2005), 1-8.

[10] M. A. Abdou and A. Elhanbaly, Construction of periodic and solitary wave solutions by the extended Jacobi elliptic function expansion method, Comm. Nonl. Sci. Num. Sim., 12 (2007), 1229-1241.

[11] M. J. Ablowitz, D. J. Kaup, A. C. Newell and H. Segur, The Inverse Scattering Transform. Fourier Analysis for Nonlinear Problems, Stud. Appl. Math., 53 (1974), 249-315.

[12] M. J. Ablowitz and P. A. Clarkson, Solitons, Nonlinear Evolution Equations and Inverse Scattering Transform, Cambridge University Press, Cambridge, 1991.

[13] M. J. Ablowitz and H. Segur, Solitons and the Inverse Scattering Transform, SIAM, 1981.

[14] G. Adomian, Nonlinear Stochastic Operator Equations, Academic Press, San Diego, (1986). 
[15] S. Antoniou, The Riccati equation method with variable expansion coefficients. I. Solving the Burgers equation, submitted for publication.

[16] S. Antoniou, The Riccati equation method with variable expansion coefficients. II. Solving the KdV equation, submitted for publication.

[17] I. Aslan, Application of the exp-function method to nonlinear lattice differential equations for multiwave and rational solutions, Math. Meth. Appl. Sci., 60 (2011), 1707-1710.

[18] D. Baldwin, Ü. Göktas, W. Hereman, L. Hong, R.S. Martino and J.C. Miller, Symbolic computation of exact solutions expressible in hyperbolic and elliptic functions for nonlinear PDEs, J. Symb. Comp., 37 (2004), 669-705.

[19] A. Bekir and A. Boz, Exact Solutions for Nonlinear Evolution Equations using Exp-Function Method, Phys. Lett. A, 372 (2008), 1619-1625.

[20] E. D. Belokolos, A. Bobenko, V. Z. Enolskii, A. R. Its and V. Matveev, Algebro-Geometric Approach to Nonlinear Integral Equations, Springer-Verlag, 1994.

[21] G. Bluman and S. Kumei, Symmetries and Differential Equations, Springer-Verlag, 1989.

[22] A. Borhanifar and A. Z. Moghanlu, Application of the $\left(G^{\prime} / G\right)$ - expansion method for the ZhiberSabat equation and other related equations, Math. Comp. Mod., 54 (2011), 2109-2116.

[23] H. T. Chen and H. Q. Zhang, Improved Jacobian elliptic function method and its applications, Chaos, Solitons and Fractals, 15 (2003), 585-591.

[24] Y. Chen and Q. Wang, Extended Jacobi elliptic function rational expansion method and abundant families of Jacobi elliptic function solutions to $(1+1)$-dimensional dispersive long wave equation, Chaos, Solitons and Fractals, 24 (2005), 745-757.

[25] A. E. Ebaid, Generalization of He's Exp-Function Method and New Exact Solutions for Burgers Equation, Z. Naturforsch, 64a (2009), 604-608.

[26] S. A. El-Wakil, E. M. Abulwafa, A. Elhanbaly and M. A. Abdou, The extended homogeneous balance method and its applications, Chaos, Solitons and Fractals, 33 (2007), 1512-1522.

[27] S. A. El-Wakil and M. A. Abdou, New exact travelling wave solutions using modified extended tanhfunction method, Chaos, Solitons and Fractals, 31 (2007), 840-852.

[28] S. A. El-Wakil, M. A. Abdou and A. Hendi, New periodic wave solutions via exp-function method, Phys. Lett., A 372, (2008), 830-840.

[29] S. A. El-Wakil and M. A. Abdou, New applications of the homotopy analysis method, Zeitschrift für Naturforschung, A (2008).

[30] E. Fan, Two new applications of the homogeneous balance method, Phys. Lett., A 265, (2000), $353-$ 357.

[31] E. Fan, Extended tanh-function method and its applications to nonlinear equations, Phys. Lett., A 277, (2000), 212-218.

[32] E. Fan and Y. C. Hon, Applications of extended tanh-method to "special types" of nonlinear equations, Appl. Math. Comp., 141 (2003), 351-358.

[33] E. Fan and H. Zhang, Applications of the Jacobi elliptic function method to special-type nonlinear equations, Phys. Lett., A 305, (2002), 383-392.

[34] J. Feng, W. Li and Q. Wan, Using $\left(G^{\prime} / G\right)$ - expansion method to seek traveling wave solution of Kolmogorov-Petrovskii-Piskunov equation, Appl. Math. Comp., 217 (2011), 5860-5865.

[35] Z. S. Feng, The first integral method to study the Burgers-Korteweg de Vries equation, J. Phys. A: Math Gen., A 302 (2002), 343-349.

[36] P. Gray and S. Scott, Chemical Oscillations and Instabilities, Clarendon Press, Oxford, 1990.

[37] P. Griffiths and W. E. Sciesser, Traveling Wave Analysis of Partial Differential Equations, Academic Press, 2012.

[38] J. H. He, A variational iteration method-a kind of nonlinear analytical technique: Some examples, Int. J. Nonl. Mech., 34 (1999), 699-708.

[39] J. H. He and M. A. Abdou, New periodic solutions for nonlinear evolution equations using expfunction method, Chaos, Solitons and Fractals, 34 (2007), 1421-1429.

[40] J. H. He and X. H. Wu, Exp-Function method for nonlinear wave equations, Chaos, Solitons and Fractals, 30 (2006), 700-708.

[41] W. Hereman and W. Malfliet, The tanh method: A Tool to Solve Nonlinear Partial Differential Equations with Symbolic Software, Available online, Colorado School of Mines.

[42] R. Hirota, The Direct Method in Soliton Theory, Cambridge University Press, 2004. 
[43] R. Hirota, Exact Solution of the KdV Equation for Multiple Collisions of Solitons, Phys. Rev. Lett., 27 (1971), 1192-1194.

[44] P. E. Hydon, Symmetry Methods for Differential Equations, Cambridge University Press, 2000.

[45] M. Inc and M. Ergüt, Periodic wave solutions for the generalized shallow water wave equation by the improved Jacobi elliptic function method, Appl. Math. E-Notes, 5 (2005), 89-96.

[46] A. Jabbari, H. Kheiri and A. Bekir, Exact solutions of the coupled Higgs equation and the Maccari system using He's semi-inverse method and expansion method, Comp. Math. Appl., 62 (2011), 21772186.

[47] A. J. M. Jawad, M. D. Petkovich and A. Biswas, Modified simple equation method for nonlinear evolution equations, Appl. Math. Comp., 217 (2010), 869-877.

[48] Y. Keskin and G. Oturanc, Reduced Differential Transform Method for Partial Differential Equations, Int. J. Nonl. Sci. Num. Sim., 10 (2009), 741-749.

[49] H Kheiri, N. Alipour and R. Dehghani, Homotopy analysis and Homotopy Padé methods for the modified Burgers-Korteweg-de Vries and the Newell-Whitehead equations, Mathematical Sciences, 5 (2011), 33-50.

[50] N. A. Kudryashov, Exact Solutions of the Generalized Kuramoto- Sivashinsky Equation, Phys. Lett., A 147 (1990), 287-291.

[51] N. A. Kudryashov, Simplest equation method to look for exact solutions of nonlinear differential equations, arXiv:nlin/0406007v1, 4 Jun 2004.

[52] N. A. Kudryashov, Nonlinear differential equations with exact solutions expressed via the Weierstrass function, arXiv:nlin/0312035v1,16 Dec., 2003.

[53] S. Liao, Homotopy Analysis Method in Nonlinear Differential Equations, Springer, 2012.

[54] S. K. Liu, Z. Fu, S. Liu and Q. Zhao, Jacobi elliptic function expansion method and periodic wave solutions of nonlinear wave equations, Phys. Lett., A 289 (2001), 69-74.

[55] S. K. Liu, Z. Fu, S. Liu and Q. Zhao, Expansion about the Jacobi Elliptic Function and its applications to Nonlinear Wave Equations, Acta Phys. Sinica, 50 (2001), 2068-2072.

[56] D. Lu and Q. Shi, New Jacobi elliptic functions solutions for the combined KdV-mKdV Equation, Int. J. Nonl. Sci., 10 (2010), 320-325.

[57] B. Q. Lu, B. Z. Xiu, Z. L. Pang and X. F. Jiang, Exact traveling wave solution of one class of nonlinear diffusion equations, Phys. Lett., A175 (1993), 113-115.

[58] W. Malfliet, Solitary wave solutions of nonlinear wave equations, Am. J. Phys., 60 (1992), 650-654.

[59] W. Malfliet, The tanh method: a tool for solving certain classes of nonlinear evolution and wave equations, J. Comp. Appl. Math., 164-165 (2004), 529-541.

[60] W. Malfliet and W. Hereman, The tanh method: I. Exact solutions of nonlinear evolution and wave equations, Phys. Scripta, 54 (1996), 563-568.

[61] W. Malfliet and W. Hereman, The tanh method: II. Perturbation technique for conservative systems, Phys. Scripta, 54 (1996), 569-575.

[62] A. Malik, F. Chand, H. Kumar and S. C. Mishra, Exact solutions of some physical models using the $\left(G^{\prime} / G\right)$ - expansion method, Pramana, 78 (2011), 513-529.

[63] B. A. Malomed, The Newell-Whitehead-Segel equation for traveling waves, arXiv:patt-sol/9605001v1.

[64] J. Murray, Mathematical Biology, Springer-Verlag, Berlin, 1989.

[65] H. Naher, F. Abdullah and M. A. Akbar, The exp-function method for new exact solutions of the nonlinear partial differential equations, Int. J. Phys. Sci., 6 (2011), 6706-6716.

[66] H. Naher, F. A. Abdullah and M. A. Akbar, New travelling wave solutions of the higher dimensional nonlinear partial differential equation by the exp-function method, J. Appl. Math., (2012).

[67] H. Naher, F. A. Abdullah and M. A. Akbar, The $\left(G^{\prime} / G\right)$ - expansion method for abundant traveling wave solutions of Caudrey-Dodd-Gibbon equation, Math. Prob. Eng., (2011).

[68] A. C. Newell and J. A. Whitehead, Finite bandwidth, finite amplitude convection, J. Fluid Mech., 38 (1969), 279-303.

[69] S. P. Novikov, S. V. Manakov, L. P. Pitaevskii and V. E. Zakharov, Theory of Solitons: The Inverse Scattering Method, Plenum, NY 1984.

[70] P. J. Olver, Applications of Lie Groups to Differential Equations, Graduate Texts in Mathematics, vol.107, Springer Verlag, N.Y. 1993.

[71] L. V. Ovsiannikov, Group Analysis of Differential Equations, Academic Press, New York, 1982.

[72] T. Ozis and I. Aslan, Application of the $\left(G^{\prime} / G\right)$ - expansion method to Kawahara type equations using symbolic computation, Appl. Math. Comp., 216 (2010), 2360-2365. 
[73] E. J. Parkes and B. R. Duffy, An automated tanh-function method for finding solitary wave solutions to nonlinear evolution equations, Comp. Phys. Comm., 98 (1996), 288-300.

[74] E. J. Parkes, B. R. Duffy and P. C. Abbott, The Jacobi elliptic function method for finding periodicwave solutions to nonlinear evolution equations, Phys. Lett., A 295 (2002), 280-286.

[75] E. J. Parkes, E. J. Zhu, B. R. Duffy and H. C. Huang, Sech-polynomial traveling solitary-wave solutions of odd-order generalized KdV equations, Phys. Lett., A 248 (1998), 219-224.

[76] K. R. Raslan, The first integral method for solving some important nonlinear partial differential equations, Nonl. Dyn., (2007).

[77] C. Rogers and W. F. Shadwick, Bäcklund Transformations, Academic Press, New York, 1982.

[78] R. G. Rojas, R. G. Elias and M. G. Clerc, Dynamics of an interface connecting a stripe pattern and a uniform state: amended Newell-Whitehead-Segel equation, Int. J. Bifurcation and Chaos, 19 (2009), 2801-2812.

[79] W. Rui, S. Xie, Y. Long and B. He, Integral Bifurcation Method and its Applications for solving the modified Equal Width Wave equation and its variants, Rostock Math. Kolloq., 62 (2007), 87-106.

[80] A. H. Salas, and C. A. Gomez, Application of the Cole-Hopf transformation for finding exact solutions to several forms of the seventh-order KdV equation, Math. Prob. Eng., (2010).

[81] L. A. Segel, Distant side-walls cause slow amplitudes modulation of cellular convection, J. Fluid Mech., 38 (1969), 203-224.

[82] A. A. Soliman and H. A. Abdou, New exact solutions of nonlinear variants of the RLW, the phi-four and Boussinesq equations based on modified extended direct algebraic method, Int. J. Nonl. Sci., 7 (2009), 274-282.

[83] H. Stephani, Differential Equations: Their Solutions Using Symmetries, Cambridge University Press, 1989.

[84] N. Taghizadeh, M. Akbari and A. Ghelichzadeh, Exact solution of Burgers equations by homotopy perturbation method and reduced differential transformation method, Austr. J. Basic Appl. Sci., 5 (2011), 580-589.

[85] N. K. Vitanov, Application of simplest equations of Bernoulli and Riccati kind for obtaining exact traveling-wave solutions for a class of PDEs with polynomial nonlinearity, Comm. Nonl. Sci. Num. Sim., 15 (2010), 2050-2060.

[86] M. L. Wang, Y. B. Zhou and Z. B. Li, Application of a homogeneous balance method to exact solutions of nonlinear equations in Mathematical Physics, Phys. Lett., A 216 (1996), 67-75.

[87] M. L. Wang and X. Z. Li, Applications of F-Expansion to periodic wave solutions for a new Hamiltonian amplitude equation, Chaos, Solitons and Fractals, 24 (2005), 1257-1268.

[88] M. Wang, X. Li and J. Zhang, The $\left(G^{\prime} / G\right)$ - expansion method and travelling wave solutions of nonlinear evolution equations in Mathematical Physics, Phys. Lett., A 372 (2008), 417-421.

[89] A.M. Wazwaz, A reliable modification of Adomian's decomposition Method, Appl. Math.Comp., 92 (1998), 1-7.

[90] A. M. Wazwaz, The tanh-coth method for solitons and kink solutions for nonlinear parabolic equations, Appl. Math. Comp., 188 (2007), 1467-1475.

[91] A. M. Wazwaz, Partial Differential Equations and Solitary Waves Theory, Springer-Verlag, Berlin Heidelberg, 2009.

[92] J. Weiss, M. Tabor and G. Carnevale, The Painlevé Property for Partial Differential Equations, J. Math. Phys., 24 (1982), 522-526.

[93] J. Weiss, M. Tabor and G. Carnevale, The Painlevé Property, J. Math. Phys., 24 (1983), $1405-$

[94] G. Whitham, Linear and Nonlinear Waves, Wiley, NY, 1974.

[95] K. Yahya, J. Biafar, H. Azari and P. R. Fard, Homotopy Perturbation Method for Image Restoration and Denoising, Available online.

[96] O. Yu. Yefimova, The modified simplest equation method to look for exact solutions of nonlinear partial differential equations, arXiv:1011.4606v1 [nlin.SI], 20 Nov., 2010.

[97] E. M. E. Zayed, Traveling wave solutions for higher dimensional nonlinear evolution equations using the $\left(G^{\prime} / G\right)$-expansion method, J. Appl. Math. Inform., 28 (2010), 383-395.

[98] E. M. E. Zayed, A note on the modified simple equation method applied to Sharma-Tasso-Olver equation, Appl. Math. Comp., 218 (2011), 3962-3964.

[99] E. M. E. Zayed and K. A. Gepreel, The $\left(G^{\prime} / G\right)$-expansion method for finding traveling wave solutions of nonlinear partial differential equations in mathematical physics, J. Math. Phys., 50 (2009), 013502. 
[100] J. Zhang, Exact and explicit solitary wave solutions to some nonlinear equations, Int. J. Theor. Phys., 35 (1996), 1793-1798.

[101] X. L. Zhang and H. Q. Zhang, A new generalized Riccati equation rational expansion method to a class of nonlinear evolution equations with nonlinear terms of any order, Appl. Math. Comp., 186 (2007), 705-714. 